\title{
Modélisation d'une dynamique de développement : le cas de l'arrondissement de Verdun à Montréal
}

\author{
Christian Jetté, Christian Paquin, Julie Chalifour et Denis Côté \\ Université de Montréal
}

\section{INTRODUCTION}

Depuis quelques années, la question du développement des communautés locales est au cœur des préoccupations des acteurs sociaux concernés par le bien-être et la santé des populations. Cette préoccupation découle d'un cheminement sociohistorique ayant pris naissance dans le cadre des réflexions et des actions menées par des praticiens, des entrepreneurs locaux et des chercheurs concernant la redéfinition de certaines pratiques de l'État-providence. Plusieurs parlent même d'un nouveau paradigme pour qualifier cette nouvelle approche qui consiste à mobiliser les forces vives des territoires locaux autour d'une stratégie de développement socioéconomique multidimensionnelle. $\mathrm{Ce}$ nouveau paradigme se démarque des stratégies plus traditionnelles en faisant du «local» l'unité géographique centrale de l'intervention, tout en interpellant une catégorie diversifiée d'acteurs sociaux. Cette catégorie inclut non seulement les acteurs provenant des secteurs public et privé, mais ceux issus également de l'entreprenariat collectif (coopératives, mutuelles et associations) et de l'action communautaire autonome.

\section{Ce nouveau paradigme se démarque des stratégies plus traditionnelles en faisant du « local » l'unité géographique centrale de l'intervention, tout en interpellant une catégorie diversifiée d'acteurs sociaux.}

Ces stratégies, souvent désignées sous le vocable de «développement local », impliquent donc la participation d'une diversité d'acteurs et mettent en relief la nécessité de s'entendre d'abord sur un certain nombre de priorités à cibler à l'intérieur des territoires concernés. L'analyse de la situation s'avère ainsi une première étape cruciale d'un processus d'action collective visant à établir une concertation active et un renouveau partenarial susceptibles d'apporter des changements durables et des réponses satisfaisantes aux besoins de communautés souvent en déclin.

C'est dans cette optique que nous avons élaboré à l'automne 2008 et l'hiver 2009, à la demande de la Concertation en développement social de Verdun, un portrait des enjeux socioéconomiques dans l'arrondissement de Verdun à Montréal. Privilégiant une approche qui mettait à profit des données à la fois qualitatives et quantitatives, nous avons voulu rendre compte le plus fidèlement possible de la situation sociale et économique qui prévalait sur le territoire. Nous avons ainsi cherché à insuffler une portée sociologique à notre travail en articulant les données statistiques recueillies, avec les informations tirées des entrevues et des tables rondes organisées avec une cinquantaine d'acteurs socioéconomiques du territoire.

Le texte qui suit met en relief certaines données du portrait socioéconomiques de Verdun que nous avons réalisé ${ }^{1}$. Après avoir fourni quelques éléments contextuels sur le territoire et son milieu, nous faisons le bilan du développement économique et de l'emploi sur le territoire au cours des dernières années. Nous nous appuyons par la suite sur ce bilan pour tenter de démontrer comment ce territoire profiterait d'une meilleure intégration des dimensions sociales et économique de son développement à travers une stratégie multisectorielle de développement local. 


\section{TROIS SOUS-TERRITOIRES FORTEMENT CONTRASTÉS}

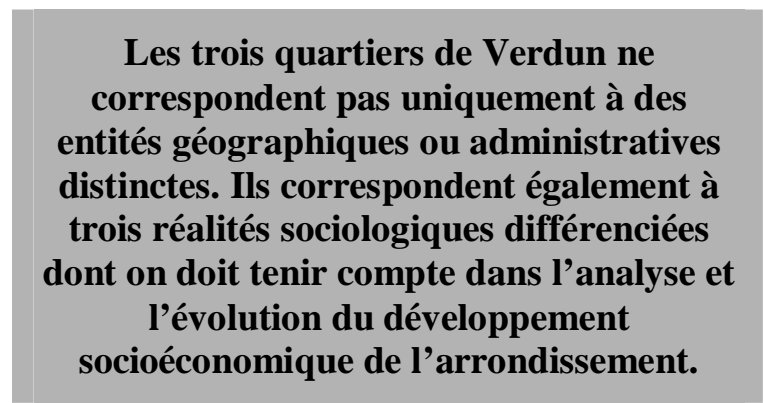

L'arrondissement de Verdun est composé de trois sous-territoires: Wellington-de-l'Église, Desmarchais-Crawford (ces deux premiers quartiers formant ce qu'on appelle la terre ferme) et l' ̂̂le-des-Sœurs. Les données statistiques révèlent que la situation socioéconomique de ces trois sous-territoires est fortement contrastée. Revenu, chômage, logement, scolarité et même architecture des bâtiments : plusieurs des indicateurs que nous avons utilisés lors de nos recherches pour analyser le territoire de Verdun ont montré des contrastes saisissants en fonction de cette subdivision du territoire, notamment lorsqu'on compare Wellington-del'Église et l'Île-des-Sœurs.

À bien des égards, on peut avancer que les trois quartiers de Verdun ne correspondent pas uniquement à des entités géographiques ou administratives distinctes. Ils correspondent également à trois réalités sociologiques différenciées dont on doit tenir compte dans l'analyse et l'évolution du développement socioéconomique de l'arrondissement.

De manière générale, ces trois sous-territoires présentent les caractéristiques suivantes. Premièrement, un centre-ville (le quartier Wellington-de-l'Église) relativement pauvre concentre une bonne part des indicateurs de défavorisation de l'arrondissement. Deuxièmement, un quartier excentré et insulaire (l' ̂̂le-desSœurs) évolue à plusieurs égards en marge de la terre ferme et accueille sur son territoire une population relativement riche et éduquée. Enfin, un quartier intermédiaire (le quartier DesmarchaisCrawford) est habité par des résidants issus de ce qu'on pourrait appeler la classe moyenne dont les caractéristiques socioéconomiques se rapprochent sensiblement des moyennes statistiques établies pour la population montréalaise dans son ensemble.

Évidemment, une analyse plus fine de chacun de ces quartiers laisserait apparaître des variations plus ou moins grandes par rapport à ce portrait, tiré à gros traits, en fonction de sousgroupes de la population ou de certains secteurs géographiques particuliers. Néanmoins, ces quelques réserves sur le portrait général de Verdun ne remettent pas pour autant en question l'interprétation générale voulant que Verdun soit constitué de trois sous-territoires fortement contrastés sur le plan socioéconomique.

\section{LE DÉVELOPPEMENT ÉCONOMIQUE ET L'EMPLOI}

L'arrondissement de Verdun a connu une période faste sur le plan de son développement social et économique allant de la fin de la Première Guerre mondiale jusqu'aux années 1960. L'essor de l'ancienne ville, fondée en 1913, s'appuyait ainsi sur la présence de nombreuses industries lourdes établies principalement dans les municipalités limitrophes: Pointe Saint-Charles, Saint-Henri, Lachine, etc. La crise qui est venue secouer cette industrie vieillissante à partir des années 1960 s'est répercutée sur le tissu socioéconomique de ces communautés et a été à l'origine du déclin observé dans toute la partie sud-ouest de Montréal, incluant Verdun.

$$
\begin{gathered}
\text { L'arrondissement de Verdun a connu une } \\
\text { période faste sur le plan de son } \\
\text { développement social et économique allant } \\
\text { de la fin de la Première Guerre mondiale } \\
\text { jusqu'aux années } 1960 .
\end{gathered}
$$


L'économie de Verdun s'est appuyée sur le développement du secteur des services, principalement dans les domaines du commerce aux détails et de la santé, notamment par la présence de deux centres hospitaliers et de quatre CHSLD sur son territoire. Ces derniers représentent aujourd'hui parmi les principaux employeurs du territoire.

Depuis, l'économie de Verdun s'est appuyée sur le développement du secteur des services (93\%), principalement dans les domaines du commerce aux détails et de la santé, notamment par la présence de deux centres hospitaliers et de quatre CHSLD sur son territoire. Ces derniers représentent aujourd'hui parmi les principaux employeurs du territoire. Selon les chiffres fournis par l'arrondissement, environ 5000 des 14000 emplois occupés par des travailleurs à Verdun relèveraient directement du secteur de la santé. Quant au secteur industriel, il fournirait $7 \%$ des emplois et serait concentré principalement à l'Île-des-Sœurs.

Un processus de revitalisation économique s'est toutefois amorcé à Verdun à partir de la seconde moitié des années 1990, sous l'égide d'un certain nombre d'acteurs préoccupés par la situation économique de l'arrondissement. Il semble que les premiers signaux d'alarme soient venus des commerçants eux-mêmes au cours des années 1980 qui ont connu une baisse sensible de leur chiffre d'affaire. À partir de là, des organismes de concertation et de mobilisation économique ont vu le jour, comme le Forum économique au début des années 1990 et le Centre local de développement un peu plus tard en 1999.

Un processus de revitalisation économique s'est toutefois amorcé à Verdun à partir de la seconde moitié des années 1990 , sous l'égide d'un certain nombre d'acteurs préoccupés par la situation économique de l'arrondissement.

Un certain nombre de promoteurs immobiliers se sont également intéressés au territoire de
Verdun à partir de la fin des années 1990 , compte tenu du coût abordable des propriétés sur la terre ferme et de la disponibilité des espaces pour la construction de nouveaux logements. L'arrondissement a également été l'instigateur d'un certain nombre d'initiatives à caractère économique. L'action de divers acteurs s'est donc conjuguée au milieu des années 1990. Elle a permis d'amorcer un regain économique sur le territoire qui s'est concrétisé par la construction de plus de 1800 nouveaux logements, la revitalisation et la diversification des artères commerciales (notamment avec une offre de produits plus haut de gamme et l'arrivée de commerçants issus des communautés culturelles) et une augmentation importante depuis 2001 de plus de $9 \%$ de la population totale de Verdun ${ }^{2}$.

\section{L'action de divers acteurs s'est donc conjuguée au milieu des années 1990. Elle a permis d'amorcer un regain économique sur le territoire.}

Ce nouvel essor économique s'est toutefois fait sentir de façon plus mitigée dans les statistiques de l'emploi, du chômage et du revenu des ménages et des particuliers et ce, même si les données recueillies montrent que le nombre de personnes occupant un emploi a augmenté de presque $13 \%$ à Verdun de 2001 à 2006, alors que ce nombre s'est accru de seulement 5,3\% dans l'ensemble de la ville de Montréal. Il faut préciser que, compte tenu de la hausse importante de la population de Verdun au cours de la même période, le taux d'emploi ne s'est accru réellement que de $2 \%$ depuis 2001.

Les questions de la faible scolarité et du décrochage scolaire expliquent en partie la situation de Verdun sur le plan de l'emploi. En général, un faible taux d'emploi chez les 15 à 24 ans résulte d'un taux de fréquentation scolaire élevée qui se reflète à son tour par un taux élevé d'emploi dans la tranche d'âge des 25 ans et plus. Cette modulation du taux d'emploi par tranche d'âge s'explique notamment par le fait qu'une scolarité plus élevée donne généralement un meilleur accès au marché du travail une fois arrivé à l'âge adulte. Comme le démontre le 
tableau 1, c'est à l' ̂̂le-des-Sœurs qu' on observe le plus faible taux d'emploi parmi le groupe des 15 à 24 ans $(39,3 \%)$, alors que le taux d'emploi pour ce même groupe d'âge s'établit à 51,6\% dans Wellington et 55,9\% dans Desmarchais. Or, qu'observe-t-on dans la tranche d'âge supérieur des 25 ans et plus ? Exactement l'inverse ! L'Île-des-Sœurs présente un taux d'emploi largement supérieur $(68,2 \%)$ à celui des deux territoires de la terre ferme qui voient, quant à eux, leur taux d'emploi augmenter d'à peine quelques points de pourcentage par rapport à la catégorie des 15 à 24 ans. L'incidence de la scolarité sur l'emploi est ici manifeste et démontre toute l'importance de la formation en tant que processus dynamique sur le développement économique et l'emploi du territoire.

\section{L'incidence de la scolarité sur l'emploi est ici manifeste et démontre toute \\ l'importance de la formation en tant que processus dynamique sur le développement économique et l'emploi du territoire.}

Tableau 1 : Indicateurs sur l'emploi en 2006

\begin{tabular}{c|cc|cc|cc}
\hline \multicolumn{8}{c|}{ Personnes employées et taux d'emploi dans la population âgée de 15 ans et plus selon l'âge } \\
& \multicolumn{2}{|c|}{15 ans et plus } & \multicolumn{2}{c}{15 à 24 ans } & \multicolumn{2}{c}{25 ans et plus } \\
& $\mathrm{Nb}$ & $\%$ & $\mathrm{Nb}$ & $\%$ & $\mathrm{Nb}$ & $\%$ \\
\hline Wellington-de-l'Église & 13240 & 53,0 & 1965 & 51,6 & 11275 & 53,2 \\
Desmarchais-Crawford & 9760 & 57,8 & 1250 & 55,9 & 8510 & 58,0 \\
\hline Terre ferme & 23000 & 54,9 & 3215 & 53,2 & 19785 & 55,2 \\
Île-des-Sœurs & 9115 & 65,6 & 505 & 39,3 & 8610 & 68,2 \\
\hline Verdun & 32115 & 57,6 & 3725 & 50,8 & 28395 & 58,6 \\
Montréal (Ville) & \multicolumn{7}{c}{57,7} & & 52,4 & & 58,7 \\
Québec & 60,4 & & 55,8 & & 61,2
\end{tabular}

Ces données sur l'emploi sont à mettre en parallèle également avec celles sur le chômage qui est passé de $8 \%$ en 2001 à $8,7 \%$ en 2006 à Verdun, soit une augmentation de $8,6 \%$ alors que pour la même période, le taux de chômage à Montréal diminuait de 5,5\%, passant de 9,7 à $9,2 \%$ (voir tableau 2). Malgré la hausse du taux de chômage à Verdun depuis 2001 (qui demeure toutefois inférieur à celui de Montréal), les possibilités d'emploi resteraient excellentes dans l'arrondissement, aux dires de certains interve- nants travaillant en employabilité que nous avons interrogés. La difficulté proviendrait davantage de l'alourdissement des problématiques avec lesquelles doivent composer les personnes en recherche d'emploi. L'amélioration générale de la situation économique (du moins, jusqu'en 2008) a eu pour conséquence paradoxale de rendre plus difficile le travail des organismes communautaires et des établissements publics travaillant dans le domaine de l'emploi (incluant le Centre local d'emploi).

Tableau 2 : Indicateurs du chômage en 2006

\begin{tabular}{|c|c|c|c|c|c|c|}
\hline \multicolumn{7}{|c|}{ Personnes en chômage et taux de chômage dans la population âgée de 15 ans et plus selon le sexe } \\
\hline & \multicolumn{2}{|c|}{ Hommes } & \multicolumn{2}{|c|}{ Femmes } & \multicolumn{2}{|c|}{ Total } \\
\hline & $\mathrm{Nb}$ & $\%$ & $\mathrm{Nb}$ & $\%$ & $\mathrm{Nb}$ & $\%$ \\
\hline Wellington-de-l'Église & 975 & 12,5 & 785 & 10,9 & 1760 & 11,7 \\
\hline Desmarchais-Crawford & 555 & 10,3 & 340 & 6,5 & 890 & 8,4 \\
\hline Terre ferme & 1530 & 11,6 & 1125 & 9,0 & 2650 & 10,3 \\
\hline Île-des-Sœurs & 155 & 3,1 & 265 & 5,9 & 420 & 4,4 \\
\hline Verdun & 1690 & 9,3 & 1380 & 8,2 & 3070 & 8,7 \\
\hline Variation 2001-2006 & 24,4 & 8,3 & 26,4 & 12,1 & 25,3 & 8,6 \\
\hline $\begin{array}{l}\text { Montréal (Ville) } \\
\text { Variation 2001-2006 (Mtl) }\end{array}$ & & 9,6 & & 8,7 & & $\begin{array}{c}9,2 \\
(5,5)\end{array}$ \\
\hline
\end{tabular}




\section{L'accès à l'emploi est donc synonyme non seulement de revenus et de consommation, mais également de socialisation et d'intégration.}

Ces derniers ont accueilli un plus grand nombre de personnes ayant un profil plus « résistant» aux programmes conventionnels de retour à l'emploi. Certaines de ces personnes connaissent des problèmes de santé mentale et de désorganisation sociale et familiale. Même constat fait par les intervenants travaillant auprès des nouveaux immigrants, une population en croissance fulgurante à Verdun puisque le nombre d'immigrants a augmenté de près de $47 \%$ depuis 2001. Mais cette fois, ce ne sont pas les problèmes d'ordre social ou de santé mentale qui freinent principalement l'accès à l'emploi (même s'ils existent là aussi), mais plutôt la non-reconnaissance des diplômes obtenus à l'étranger par plusieurs ordres professionnels, ainsi que le maintien de pratiques discriminatoires à l'embauche, pratiques qui perdurent même auprès des membres des communautés ethnoculturelles issus des deuxième et troisième générations. Or, cette problématique de l'emploi a des répercussions sur le plan social puisque, comme l'affirmait une intervenante travaillant auprès des communautés culturelles : « la question de l'emploi est fondamentale. [...] C'est le nerf de la guerre de l'intégration. Quelqu'un qui n'a pas d'emploi ne pourra pas s'intégrer. » L'accès à l'emploi est donc synonyme non seulement de revenus et de consommation, mais également de socialisation et d'intégration.
Par ailleurs, les données sur les revenus des particuliers dans l'arrondissement laissent entrevoir, comme on pouvait s'y attendre, des disparités importantes selon les territoires.

Sur la terre ferme, la moyenne de revenus annuels des particuliers s'établissait en 2005 à 25591 \$, soit une différence inférieure de près de $5000 \$$ à la moyenne montréalaise (30 117 \$). À l'île-des-Soeurs, la moyenne des revenus annuels s'élevait, quant à elle, à près de 69000 \$. Ces données statistiques tendent ainsi à montrer que le regain économique qu'a connu Verdun depuis une dizaine d'années ne connaît pas la même intensité et n'a pas les mêmes répercussions sur l'ensemble des trois territoires (voir tableau 3). Cette observation a été corroborée par plusieurs personnes ayant participé aux tables rondes que nous avions organisées et qui reconnaissent qu'il y a bien eu un essor économique à Verdun, notamment sur le plan immobilier, mais que cet essor n'a pas profité de manière équivalente à tous les citoyens. Selon certains intervenants, les inégalités socioéconomiques persistent et se creusent même dans certains cas.

\section{Le regain économique qu'a connu Verdun depuis une dizaine d'années ne connaît pas la même intensité et n'a pas les mêmes répercussions sur l'ensemble des trois territoires.}

Tableau 3 : Indicateurs sur les revenus en 2005

\begin{tabular}{|c|c|c|c|c|c|c|}
\hline \multicolumn{7}{|c|}{ Revenu total moyen des particuliers de 15 ans et plus } \\
\hline \multirow{3}{*}{ Territoire } & \multirow{3}{*}{$\begin{array}{c}\text { Total } \\
\$\end{array}$} & \multirow{3}{*}{$\begin{array}{c}\text { Hommes } \\
\$\end{array}$} & \multirow{3}{*}{$\begin{array}{c}\text { Femmes } \\
\$\end{array}$} & \multicolumn{3}{|c|}{ Évolution 2000-2005 } \\
\hline & & & & Total & Hommes & Femmes \\
\hline & & & & $\%$ & $\%$ & $\%$ \\
\hline Wellington-de-l'Église & 23003 & 24332 & 21745 & 12,6 & 4,3 & 23,0 \\
\hline Desmarchais-Crawford & 29423 & 33301 & 26087 & 15,2 & 13,2 & 16,6 \\
\hline Terre ferme & 25591 & 27842 & 23542 & 13,4 & 8,1 & 19,3 \\
\hline Île-des-Sœurs & 68901 & 87511 & 52325 & 8,2 & 8,3 & 8,1 \\
\hline Verdun & 36411 & 42620 & 30789 & 14,8 & 12,1 & 17,9 \\
\hline Montréal (Ville) & 30117 & 34532 & 26038 & 15,8 & 13,7 & 18,5 \\
\hline Québec & 32074 & 38509 & 25870 & 18,2 & 16,3 & 21,5 \\
\hline
\end{tabular}


Le développement endogène ne doit pas reposer uniquement sur l'arrivée de nouveaux résidants venus s'installer à la faveur d'opportunités intéressantes sur le plan immobilier, ou d'une mise en valeur des avantages « naturels » de

l'arrondissement (présence du fleuve, proximité du centre-ville).

En résumé, s'il est indéniable que Verdun a progressé de manière importante sur le plan économique au cours des dernières années, d'énormes défis restent encore à relever afin d'assurer un développement qui soit davantage endogène à sa communauté.

Le développement endogène ne doit pas reposer uniquement sur l'arrivée de nouveaux résidants venus s'installer à la faveur d'opportunités intéressantes sur le plan immobilier, ou d'une mise en valeur des avantages «naturels» de l'arrondissement (présence du fleuve, proximité du centre-ville). Certes, cet apport de sang neuf au sein de la population ne peut être que bénéfique à l'arrondissement, non seulement en raison de l'apport économique qu'elle entraîne, mais en raison également de la diversification sociale et culturelle qu'il procure à une communauté souvent perçue comme homogène par les personnes mêmes que nous avons interrogées.

Mais ce développement doit également se réaliser par l'amélioration des conditions de vie d'une partie de la population qui est encore loin de profiter pleinement des retombées inhérentes à ce nouveau dynamisme insufflé à l'arrondissement. À cet égard, la question du décrochage scolaire et du faible taux de scolarité d'une partie de la population verdunoise nous est apparue à moyen terme comme une menace directe à la poursuite du développement social et économique de l'arrondissement. Cette problématique ne peut venir qu'alimenter les disparités observées sur les plans socioéconomique et territorial, et venir entacher la cohésion sociale d'une communauté qui, jusqu'à présent, a su faire preuve d'un fort sentiment d'attachement à son territoire.
À cet égard, nous avons souligné un peu plus haut le dynamisme socioéconomique engendré par l'augmentation d'un peu plus de $9 \%$ de la population dans l'arrondissement. Mais encore faut-il convaincre ces nouveaux résidants de vivre au quotidien à Verdun, et même de consommer à Verdun puisque pour le moment, il semble qu'une bonne proportion d'entre eux continue d'avoir des habitudes de vie qui les amènent à consommer principalement à l'extérieur de l'arrondissement. Ce qui nous ramène à la nécessité, pour l'ensemble des acteurs socioéconomiques de Verdun, de travailler sur des stratégies locales de développement qui pourraient être orientées d'une part, vers une meilleure intégration de ces nouveaux résidants et, d'autre part, vers le relèvement des conditions de vie des résidants moins fortunés, souvent établis de longue date, et qui, à plusieurs égards, se sont maintenus en marge des transformations qui ont marqué Verdun depuis le milieu des années 1990.

Ainsi, compte tenu des enjeux qui se profilent à Verdun, il est raisonnable de croire que ces nouvelles stratégies devront être en mesure d'assurer une meilleure articulation des aspects sociaux et économiques du développement de l'arrondissement. Or, les propos recueillis auprès de nos informateurs montrent que, jusqu'à maintenant, ces deux dimensions ont plutôt été considérées comme des entités presque antinomiques, du moins difficilement conciliables dans la pratique au quotidien des acteurs identifiés à l'une ou à l'autre de ces dimensions dont l'analyse fait l'objet de la prochaine section.

Compte tenu des enjeux qui se profilent à Verdun, il est raisonnable de croire que ces nouvelles stratégies devront être en mesure d'assurer une meilleure articulation des aspects sociaux et économiques du développement de l'arrondissement. 


\section{POUR UNE MEILLEURE INTÉGRATION DES DIMENSIONS SOCIALES ET ÉCONOMIQUES DU DÉVELOPPEMENT}

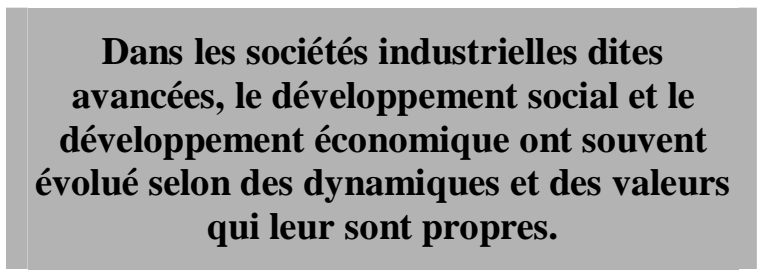

Si nous avions à résumer en une seule phrase la situation entourant cette problématique dans l'arrondissement de Verdun, nous citerions cet acteur qui affirmait en entrevue que «le développement économique et le développement social, c'est presque comme deux réalités qui s'affrontent » à Verdun. Cette coupure entre le social et l'économie n'est pas nouvelle et n'est pas exclusive à l'arrondissement de Verdun. Dans les sociétés industrielles dites avancées, le développement social et le développement économique ont souvent évolué selon des dynamiques et des valeurs qui leur sont propres. Ils sont le plus souvent portés par des acteurs différents qui se côtoient rarement ou sporadiquement, sinon pour s'affronter sur les stratégies à adopter, quand ce n'est pas sur l'orientation même à donner au développement. Ces acteurs trouvent ainsi peu d'espace de dialogue pour établir des liens favorisant l'établissement de compromis dans le cadre d'une approche plus globale du développement des communautés. Ce «désencastrement » du social et de l'économique s'est ainsi avéré une caractéristique prégnante des économies de marché, malgré les mesures prises historiquement par l'État-providence, notamment par le biais de ses politiques sociales, pour maintenir un certain équilibre systémique entre ces deux grands pôles de développement ${ }^{3}$.

Or, les crises successives qui ont frappé les économies développées depuis les années 1980 ont provoqué une remise en question du modèle de développement tel que nous l'avions connu depuis la Seconde Guerre mondiale. Cette remise en question a pris des couleurs différentes selon les lieux et les époques. Si certains ont profité de ces remises en question pour affirmer (et appliquer) avec encore plus de conviction leur croyance en un modèle axé sur l'essor d'une économie de marché libérée de toutes entraves sociales et politiques (le modèle néolibéral), d'autres - dont on peut penser que le Québec fait partie, du moins dans certaines de ses composantes locales - ont misé sur le fait qu'il était possible de développer une économie de marché à visage plus humain, sur les bases d'une plus grande justice sociale, et davantage ancrée dans ses composantes sociocommunautaires et politiques. Ce nouveau «modèle de développement » (qui est toutefois loin d'avoir connu une application généralisée sur l'ensemble du territoire québécois) a pu voir le jour grâce à l'émergence de nouveaux acteurs - par exemple, les Corporations de développement économique communautaire, les Centres locaux de développement et les entreprises d'économie sociale - et repose sur l'expérimentation de nouvelles façons de faire (qu'on pourrait qualifier d'innovations sociales) se déployant le plus souvent sur le plan local. Cette référence au local ${ }^{4}$ n'est pas un hasard. Elle s'est imposée dans un contexte de persistance d'importantes inégalités socioéconomiques dans différents territoires et dans certaines communautés locales dont la situation s'était peu améliorée (voire avait pu se détériorer) malgré l'application de diverses mesures, programmes et politiques centralisés et adoptés par l'État-providence sur le plan national au cours de la période précédente ${ }^{5}$.

Cette nouvelle façon de faire, souvent désignée sous le vocable de «développement local » se caractérise par une action intersectorielle de concertation et de partenariat (qui peut parfois s'avérer aussi conflictuelle) et vise à apporter des solutions aux problèmes globaux ou spécifiques de développement socioéconomique vécus par une population établie sur un territoire local ou régional. Cette prise en charge du développement par les acteurs locaux - avec le soutien plus ou moins grand de l'État central - a fait l'objet de nombreuses études depuis une vingtaine d'années au Québec, au Canada et ailleurs dans le monde ${ }^{6}$. Le tableau 4 présente les principales caractéristiques des trois modèles que nous venons d'évoquer. 
Tableau 4 : Caractéristiques des trois modèles de développement

\begin{tabular}{|c|c|c|c|}
\hline & Néolibéral & État-providence & Local et solidaire \\
\hline Régulation & $\begin{array}{l}\text { Libre marché (planification } \\
\text { minimale) }\end{array}$ & Planification centralisée & $\begin{array}{l}\text { Planification concertée et } \\
\text { partenariale (social + } \\
\text { économie) }\end{array}$ \\
\hline $\begin{array}{l}\text { Type de } \\
\text { politique }\end{array}$ & $\begin{array}{l}\text { Dérèglementation et } \\
\text { privatisation }\end{array}$ & $\begin{array}{l}\text { Politiques standardisées } \\
\text { sur l'ensemble du territoire }\end{array}$ & $\begin{array}{l}\text { Politiques adaptées aux } \\
\text { territoires locaux et } \\
\text { régionaux (développement } \\
\text { local) }\end{array}$ \\
\hline $\begin{array}{l}\text { Principe } \\
\text { d'action }\end{array}$ & Concurrence & Redistribution & $\begin{array}{l}\text { Actions intersectorielles et } \\
\text { plurielles }\end{array}$ \\
\hline $\begin{array}{l}\text { Acteurs } \\
\text { principaux }\end{array}$ & Entreprise privée & $\begin{array}{l}\text { Bureaucratie étatique et } \\
\text { entreprise privée }\end{array}$ & $\begin{array}{l}\text { Entreprises privées + } \\
\text { entreprises d'économie } \\
\text { sociale + institutions } \\
\text { publiques }\end{array}$ \\
\hline
\end{tabular}

Ainsi, nous nous sommes permis un bref retour historique sur l'évolution des principales dynamiques de développement socioéconomique qui animent notre société à l'heure actuelle afin de situer dans une perspective plus large les enjeux entourant la situation socioéconomique à Verdun. Cette situation, comme nous l'ont rapportée plusieurs intervenants, s'apparente ainsi - ou du moins s'apparentait jusqu'à tout récemment — à une vision plutôt traditionnelle du développement, c'est-à-dire une vision où domine un cloisonnement assez étanche entre les stratégies mises de l'avant par les acteurs du développement social et ceux du développement économique. Ces pratiques «en circuits fermés » ne concernent pas uniquement les rapports existant entre les acteurs des milieux sociaux et économiques dans l'arrondissement. Elles marquent également à divers degrés les rapports unissant les acteurs du développement social entre eux, ainsi que ceux du développement économique qui ont tendance, les uns comme les autres, à organiser leurs actions sur des bases sectorielles. En outre, - et peut-être parce que jusqu'en 2001, Verdun était encore une ville - les acteurs tant sociaux qu'économiques de l'arrondissement semblent avoir de la difficulté à s'ouvrir à des concertations élargies intégrant des acteurs provenant de l'extérieur du territoire. Aux dires d'un intervenant, "Verdun est une addition (d'acteurs) fonctionnant très bien entre eux, mais s'ignorant mutuellement ou se regardant avec politesse et s'invitant parfois ». Le fonctionnement «en silo » amène les acteurs des milieux sociaux et économiques à entretenir certains préjugés les uns envers les autres, ce qui n'est pas de nature à favoriser d'éventuels collaborations ou compromis.

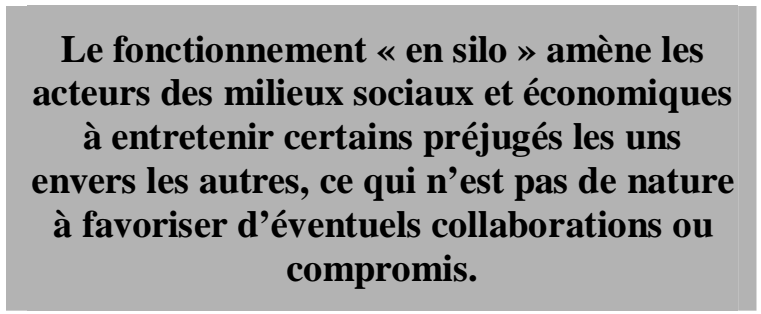

Sur le plan stratégique, le rapprochement des acteurs du développement social et du développement économique semble pourtant constituer un enjeu fondamental pour le développement de Verdun. Selon un des acteurs que nous avons interrogés, «ça serait un défi de trouver des ponts entre le développement social et le développement économique. Les deux ne s'opposent pas, les deux ont des divergences dans les moyens, dans la culture, dans la façon de s'habiller, dans les façons d'entrevoir les choses. (Mais) la journée (...) où on respecte(ra) un peu nos différentes idées, nos façons de faire, ça aura une portée incroyable. » Ce point de vue semble partagé par plusieurs personnes qui soulignaient, en entrevue, la nécessité de regrouper les forces vives de l'arrondissement. Des expressions telles "agir ensemble », «bouger ensemble », «faire ensemble», «passer à l'action»sont revenues fréquemment dans les propos des acteurs que nous avons rencontrés. Ces témoignages tendent à démontrer que plusieurs acteurs sont prêts à s'investir «autrement» dans les concertations. Il est désormais temps de dépasser le stade initial de la concertation qui 
consistait principalement à échanger de l'information ou à établir des collaborations limitées à du soutien ponctuel pour la réalisation de projets initiés par l'un ou l'autre des acteurs concernés. Une plus grande collaboration entre les acteurs des milieux sociaux et économiques est possible afin de dépasser les limites de leurs actions respectives et ainsi d'insuffler un nouveau dynamisme au processus de développement dans la communauté.

\section{Une plus grande collaboration entre les acteurs des milieux sociaux et économiques est possible afin de dépasser les limites de leurs actions respectives et ainsi d'insuffler un nouveau dynamisme au processus de développement dans la communauté.}

Certains intervenants ont mentionné qu'il avait probablement été nécessaire, dans un premier temps, de se concerter sectoriellement. Les milieux communautaires, notamment, ont trouvé là un espace de consolidation et de reconnaissance de leurs actions. La situation actuelle exige toutefois de passer à une étape subséquente qui implique de travailler davantage dans un espace multisectoriel. Cette nécessité proviendrait non seulement de la situation des acteurs socioéconomiques de Verdun, plus conscients de l'importance de franchir une nouvelle phase de l'évolution de leurs pratiques de concertation, mais en raison également des pressions extérieures, notamment des bailleurs de fonds et des instances politiques qui rendent désormais leurs appuis conditionnels à l'établissement de véritable partenariat pour la réalisation de projets structurant pour les communautés. Il y a donc, pour reprendre les termes d'une intervenante, « une question d'alignement des planètes » qui fait en sorte de favoriser le rapprochement des acteurs sociaux et des acteurs économiques sur le territoire.

Cette question est d'autant plus cruciale que plusieurs tables de concertation sectorielles du territoire semblent éprouver des difficultés. Démobilisation du membership, perte de subventions, lourdeur de fonctionnement : certains, comme la Table de sécurité alimentaire de Verdun, en sont arrivés à la conclusion «qu'il n'y a pas d'argent pour financer la concertation, il faut des projets concrets ». Même constat de la part de la Table de concertation jeunesse de Verdun (6-17 ans) et de la Table de concertation sur le développement culturel qui ont l'impression de «tourner en rond» et qui se cherchent un projet mobilisateur afin de dynamiser davantage leur action. Bref, même si plusieurs acteurs réaffirment leur besoin de se concerter, ils désirent le faire «autrement», « en faisant preuve de créativité et d'audace » et en étant davantage «interpellés par les enjeux du quartier ».

Certaines tables de concertation ont donc amorcé une réflexion sur leurs modes de fonctionnement et les objectifs de leurs actions. Cette réorientation implique à bien des égards la mise en place d'actions intersectorielles et, conséquemment, l'établissement de ponts avec les milieux économiques. Ce rapprochement est d'ailleurs déjà commencé dans certains secteurs. De par leurs activités, certains acteurs des milieux sociaux et économiques ont été amenés à travailler plus étroitement au cours des derniers mois. Soulignons entre autres les activités entourant la sécurité alimentaire ou la question du logement social. Selon certains, une transformation des mentalités est en train de s'opérer dans l'arrondissement qui fait en sorte que les acteurs sociaux et économiques peuvent maintenant «avancer dans la saine confrontation », et non plus se retrancher derrière des positions suscitant méfiance et opposition réciproques. D'ailleurs, ce ne sont pas tant les objectifs visés par leur action qui semblent faire problème auprès des différents acteurs. Tous les acteurs s'entendent en général sur la nécessité de travailler au progrès socioéconomique de Verdun ainsi qu'à l'amélioration du mieux-être collectif de sa population. C'est plutôt sur les façons de faire que «ça accroche», pour reprendre l'expression d'un acteur interrogé.

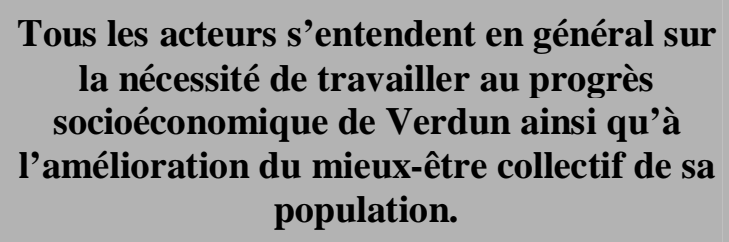


Mais pour passer de la parole aux actes, cette volonté exprimée par plusieurs «d'agir ensemble » devra se concrétiser au sein de projets bien réels en mesure de susciter une adhésion forte de la part des acteurs locaux. Car ce n'est qu'à cette condition qu'il sera possible d'amorcer un processus de développement qui soit davantage systémique, c'est-à-dire un processus où les dynamiques de développement social et de développement économique se soutiennent réciproquement et viennent se renforcer mutuellement.

Selon Klein, un chercheur bien connu au Québec dans le domaine du développement local, l'une des premières conditions de réalisation d'un tel cycle vertueux de développement est l'implication des acteurs autour d'une initiative locale, c'est-à-dire d'un projet individuel ou collectif qui mène à la réalisation d'un partenariat entre les acteurs locaux. Certes, ce type de projet provoque souvent au départ des réactions mitigées, voire de l'opposition de la part de certains acteurs issus de l'intérieur ou de l'extérieur de la collectivité. L'enjeu devient alors de susciter suffisamment d'intérêt autour de ce projet afin que tous les acteurs concernés puissent débattre de sa pertinence et, ultimement, fassent les compromis nécessaires à sa réalisation. À plus ou moins long terme, cette nouvelle solidarité autour d'une initiative locale amène les acteurs «à mettre l'accent sur ce qui les unit, soit l'appartenance territoriale commune, et à nuancer ce qui les sépare ${ }^{7}$. Les acteurs se comportent ainsi comme des «entrepreneurs collectifs » qui peuvent cristalliser leur expérience commune grâce à diverses instances organisationnelles, lesquelles pourront éventuellement être remises en tension lors de la réalisation de nouveaux projets.

\section{Les acteurs se comportent ainsi comme des « entrepreneurs collectifs » qui peuvent cristalliser leur expérience commune grâce à diverses instances organisationnelles, lesquelles pourront éventuellement être remises en tension lors de la réalisation de nouveaux projets.}

À la lumière de ces brèves considérations sur le développement local, on comprend que la cohésion des divers acteurs socioéconomiques va constituer un enjeu majeur à Verdun au cours des prochaines années. Comme l'affirmait en entrevue un intervenant travaillant dans le domaine de la sécurité alimentaire, «on doit essayer de réfléchir ensemble à comment coordonner nos efforts en intervention de sécurité alimentaire pour répondre à un modèle global qui essaie de tenir compte de plus de dimensions que juste celles qu'on atteint dans nos organismes ». Ce principe de coordination auquel fait référence cet intervenant pourrait ainsi s'appliquer à bien d'autres domaines d'intervention. Il s'agit d'un principe général orienté vers une action intersectorielle et concertée qui vise précisément à désenclaver l'action de chacun des acteurs et à se rapprocher davantage d'un modèle intégré de développement.

Le sentiment d'appartenance à un territoire s'avère un formidable réservoir d'initiatives et de dynamisme sur le plan local qui se nourrit, à son tour, des succès de l'action concertée des acteurs locaux.

À cet égard, Verdun possède des atouts indéniables. Comme nous l'avons souligné précédemment, le sentiment d'identification et d'appartenance au territoire est très fort dans l'arrondissement (du moins, pour la population de la terre ferme). Or, pour Klein comme pour d'autres chercheurs du développement local, cette question de l'appartenance s'avère cruciale dans le développement d'une communauté. René Lachapelle, un organisateur communautaire ayant une longue expérience de mobilisation auprès des communautés ${ }^{8}$, affirme : «Il n'y a pas de mobilisation sans appartenance... la proximité géographique sur un territoire est un facteur déterminant pour l'établissement de rapports d'entraide dans lesquels les gens se reconnaissent et développent la capacité de se prendre en charge et de prendre en charge leur milieu ${ }^{9}$. Ainsi, le sentiment d'appartenance à un territoire s'avère un formidable réservoir d'initiatives et de dynamisme sur le plan local qui se nourrit, à son tour, des succès de l'action concertée des acteurs locaux. 
Un cercle vertueux est généré en tant que fruit d'un processus enclenché par les acteurs euxmêmes, lequel s'avère propice à l'établissement de liens constructifs entre le social et l'économique. L'amorce de ce processus exige toutefois l'établissement de compromis de la part des différents acteurs qui ont souvent lutté entre eux pour l'obtention des ressources et la

\section{CONCLUSION}

\section{L'arrondissement de Verdun dispose d'indéniables atouts qui lui permettent d'aspirer à une redynamisation de son tissu social et économique.}

Notre étude sur les enjeux socioéconomiques de Verdun laisse apparaître un arrondissement caractérisé par d'importantes disparités socioéconomiques et territoriales. Les données recueillies ont fait ressortir les discontinuités sociales, économiques et culturelles qui marquent les limites de chacun des trois quartiers qui composent l'arrondissement. Mais en dépit de ce développement fortement contrasté, hérité principalement du déclin ayant marqué Verdun depuis les années 1960, l'arrondissement de Verdun dispose d'indéniables atouts qui lui permettent d'aspirer à une redynamisation de son tissu social et économique. Cette ancienne ville, fusionnée avec Montréal en 2001, peut ainsi tabler sur une population fortement attachée à son territoire, ainsi que sur des acteurs socioéconomiques disposant d'un capital important en termes de ressources, d'énergie et de liens sociaux. Les efforts faits depuis une dizaine d'années pour relancer son développement rendent compte de la vitalité et de la confiance que manifestent ces acteurs envers leur communauté.

Néanmoins, tout en s'étant améliorée au cours des dernières années, la situation socioéconomique prévalant à Verdun demeure sous bien des aspects désavantagée par rapport à Montréal ou au reste du Québec. Cela signifie que les acteurs du milieu devront déployer des efforts importants pour apporter des correctifs à une situation qui perdure depuis maintenant près reconnaissance sociale dans l'arrondissement. La question devient alors de savoir si les acteurs du développement social et économique de Verdun sont prêts à dépasser ces vieux clivages et à établir de nouvelles formes de collaboration. Évidemment, la réponse à cette question leur appartient...

d'un demi-siècle et qui commande, pour y remédier, de trouver de nouvelles stratégies d'intervention. À cet égard, plusieurs acteurs de l'arrondissement de Verdun se sont entendus sur la nécessité de procéder à une meilleure intégration des dimensions sociales et économiques du développement à l'intérieur de l'arrondissement. Verdun est desservi par une multitude de tables de concertation sectorielles, d'organismes communautaires, de regroupements d'affaires et d'institutions publiques.

\section{plusieurs acteurs de l'arrondissement de Verdun se sont entendus sur la nécessité de procéder à une meilleure intégration des dimensions sociales et économiques du développement à l'intérieur de l'arrondissement}

Ces différents acteurs ont toutefois eu tendance — jusqu'à tout récemment du moins — à intervenir en rangs dispersés, tout en se manifestant une méfiance réciproque. Cette méfiance se fait particulièrement sentir entre les acteurs issus des milieux sociaux et ceux des milieux économiques. À la lumière des données et des témoignages recueillis, nous pensons que les vieux réflexes sectoriels et les guerres de clocher doivent être mis de côté afin de laisser place à de nouvelles formes d'intervention plus partenariales et multisectorielles. 


\section{Bibliographie}

${ }^{1}$ Jetté, C. et Paquin, C. avec la collaboration de Chalifour, J. et Côté, D. (2009). Agir ensemble à Verdun. Portrait et enjeux socioéconomiques du territoires, Montréal, LAREPPS et CDSV, 153 p. Le document est disponible à l'adresse suivante : www.larepps.uqam.ca.

${ }^{2}$ En 2006, la population de Verdun s'élevait à un peu plus de 66000 habitants.

${ }^{3}$ Polanyi, K. (1983). La Grande Transformation, Paris, Gallimard, $419 \mathrm{p}$.

${ }^{4}$ Hamel, P. et Silvestro, M. (2005). « Mouvements urbains et démocratisation des politiques urbaines dans le contexte de la mondialisation », dans Guay, L., Hamel, P., Masson, D. et Vaillancourt, J.-G., Mouvements sociaux et changements institutionnels. L'action collective à l'ère de la mondialisation, Québec, PUQ, pp. 91-113.

${ }^{5}$ Favreau, L. (2008). Entreprises collectives. Les enjeux sociopolitiques et territoriaux de la coopération et de l'économie sociale, Québec, PUQ, 332 p.

${ }^{6}$ Laville, J.-L., Magnen, J.-P., de França, F., Genauto C. et Medeiros, A. (2005). Action publique et économie solidaire. Une perspective internationale, Paris, Éditions Érès, 414 p.

${ }^{7}$ Klein, J.-L. (2006). « Développement local et initiative locale : Une perspective d'analyse et d'intervention », dans Tremblay, M., Tremblay, P.-A. et Tremblay, S. Le développement social. Un enjeu pour l'économie sociale, Québec PUQ, pp. 162-175 (p.156).

${ }^{8}$ René Lachapelle fut président du Regroupement québécois des intervenants et intervenantes en action communautaire en CLSC et en Centre de santé (RQIIAC) de 2002 à 2006. Il occupe actuellement le poste de secrétaire général du Groupe d'économie solidaire du Québec (GESQ).

${ }^{9}$ Lachapelle, R. (2006). «Retrouver notre capacité d'initiative. Le développement des communautés et l'État libéral », dans Tremblay, M., Tremblay, P.-A. et Tremblay, S. Le développement social. Un enjeu pour l'économie sociale, Québec PUQ, pp. 150-161. 